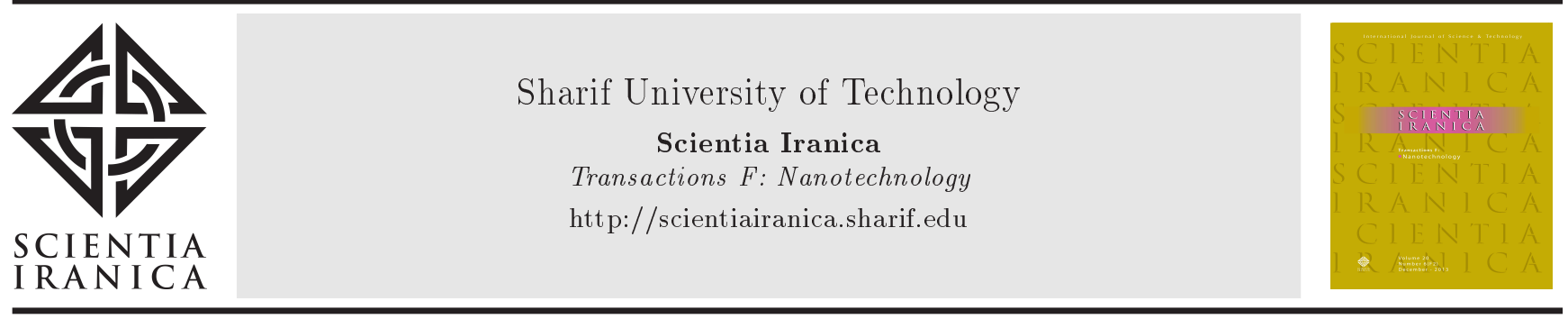

\title{
Entropy generation analysis of peristaltic flow of magneto-nanoparticles suspended in water under second-order slip conditions
}

\author{
T. Hayat ${ }^{a, b}$, S. Nawaz ${ }^{a, *}$, and A. Alsaedi ${ }^{b}$ \\ a. Department of Mathematics, Quaid-I-Azam University 45320, Islamabad 44000, Pakistan. \\ b. Nonlinear and Applied Mathematics (NAAM) Research Group, Department of Mathematics, King Abdulaziz University, Jeddah \\ 21589, Saudi Arabia.
}

Received 10 October 2019; received in revised form 23 December 2019; accepted 18 February 2020

\author{
KEYWORDS \\ $\mathrm{Fe}_{3} \mathrm{O}_{4}$-water \\ nanofluid; \\ Peristalsis; \\ Mixed convection; \\ Second-order velocity \\ and thermal slip \\ conditions; \\ Entropy generation; \\ MHD.
}

\begin{abstract}
Peristalsis of magneto-nanoparticles suspended in water was investigated in this study. $\mathrm{Fe}_{3} \mathrm{O}_{4}$-water nanofluid was utilized for two-dimensional flow in channel, which was considered symmetric with compliant walls. Uniform magnetic field was applied and temperature equation was arranged for viscous dissipation. Also, second-order velocity and thermal slip conditions were utilized. Small Grashof number led to the perturbation solution. Examination of entropy generation was also carried out. Maxwell and HamiltonCrosser models was used. The analysis was based on the comparative study of these two models representing the cylindrically and spherically shaped particles. Graphs for velocity, temperature, entropy generation, and Bejan numbers were plotted under the influence of sundry variables. Trapping was observed via plotting streamlines.
\end{abstract}

(C) 2020 Sharif University of Technology. All rights reserved.

\section{Introduction}

Nanotechnology has attracted the attention of recent researchers due to its ample applications to industrial, biomedical, and engineering fields. It has made many advancements in our daily life. For instance, through the use of nanoparticles, we can remove the harmful and dangerous viruses and bacteria from water. Vehicle fuel efficiency and corrosion resistance can also be improved by making the vehicle parts from nanocomposite materials. These materials can also be employed to produce sports goods with features such as higher strength, light weight, etc. The term

*. Corresponding author. Tel.: +92 5190642172

E-mail address: sadafnawaz@math.qau.edu.pk (S. Nawaz)

doi: $10.24200 /$ sci.2020.54615.3835 nanofluid was first used by Choi [1]. Nanomaterials are very useful materials with particle sizes in the nano $\left(10^{-9}\right.$ meter) scale. Nanofluids are made by the mixture of a base fluid, e.g., water, oil, ethylene glycol, etc., and nanoparticles. Nanoparticles have different shapes, e.g., spherical, cylindrical, tube, blade, bricks, etc., and various types including the oxides, carbides, nanotubes, etc. Different two-phase models have been utilized by researchers in the literature. In two-phase models for the nanofluids, thermo-physical characteristics of the nanomaterial and base liquid are separately defined $[2,3]$. These models provide the advantage of investigating various types of nanofluids by using different nanoparticles and base fluids, hence the capability to make a comparison among them for identifying the ones with better performances. On the other hand, in single-phase models, like Buongiorno model [4], thermo-physical properties of each fluid are 
not specified. Thus, one can analyze the fluid in general by considering the prominent effects of nanofluid, i.e. Brownian motion and thermophoresis effects. Favorable thermo-physical properties of nanofluid have drawn the curiosity of researchers and have led to many advancements in engineering, biomedical, and industrial fields, hence being a hot topic of research. Mahanthesh et al. [5] investigated a two-phase model for nanofluids by utilizing the SWCNT and MWCNT in which the flow was made by a rotating disk. Hsiao [6] examined the stretching sheet problem for micropolar nanofluid. Makinde et al. [7] discussed the flow made by a rotating disk in the presence of aluminum and titanium alloy nanoparticles. Some other studies in the literature dealing with nanofluids can be found in [813], which shed more light on the utility of nanofluids.

In physiology, bulk fluid movement is due to the peristaltic phenomenon. In this mechanism, a progressive wave moves within the channel walls, forcing the fluid to flow. This phenomenon is significantly at work in human body. Some obvious instances are the transportation of food from esophagus, movement of chyme, transportation of urine to bladder, transportation of lympth in lymphatic vessels, etc. In addition to physiological processes, peristalsis are significant in industrial processes and biomedical applications. Modern devices like dialysis machine, open heart bypass machine, etc. operate under peristalsis. This principle is useful for transferring corrosive fluids since it prevents their contamination with machinery. Hence, peristalsis is applicable to sanitary fluid transportation. Many engineering devices like hose pump, roller pump, finger pump, etc. operate on the same principle. The broad physiological, biomedical, and industrial applicability of peristalsis make them a significantly important area of research. Thus, many research works on peristalsis have been carried out after the pioneering research of Latham [14], e.g. [15-27]. Peristaltic transport of nanofluids can be consulted by many attempts in [28$36]$.

It is well known that several aspects such as chemical reaction, Joule heating, etc. in a thermodynamic system affect its entropy. Basically, the entropy of a system is a measure of disorder in it. Bejan [37] presented the first analysis of entropy. The existing literature provides insufficient information on peristaltic transportation of fluid with entropy (see [3840]). Hence, the main objective of the present study is to further investigate this regime. Peristalsis of nanofluid subject to entropy generation under mixed convection is considered. $\mathrm{Fe}_{3} \mathrm{O}_{4}$ with water as the base fluid is used as the nanofluid. There is no study to deal with the second-order slip conditions in terms of both velocity and temperature and this study is aimed to fill this gap. Specifically, the HamiltonCrosser and Maxwell models are comparatively used for the cylindrical and spherical particles, respectively. The resulting problems employing lubrication approach are solved by regular perturbation method. Grashof number is adopted as the perturbation quantity. Velocity, temperature, entropy generation, and Bejan number are sketched and examined. Trapping phenomenon is studied by graphical illustration of streamlines.

\section{Flow configuration}

Peristaltic flow of an incompressible nanofluid composed of $\mathrm{Fe}_{3} \mathrm{O}_{4}$ and water is considered. The channel (with the width of $2 d$ ) is considered symmetric. Flexible channel walls are placed at the positions $y=$ $\pm \eta$, where the left and right walls are denoted by and + , respectively (see Figure 1 ). The rectangular coordinates system is set such that the $x$-axis lies in the direction of channel length and the $y$-axis is perpendicular to it. Temperature of the walls is maintained at $T_{0}$. Contribution of the applied constant magnetic field is taken into account. Induced magnetic and electric fields effects are omitted. Mixed convection and viscous dissipation are studied. Sinusoidal wave has the wavelength of $\lambda$, amplitude of $a$, and speed of $c$. The shape of the wave is defined by the equation given below:

$$
y= \pm \eta(x, t)= \pm\left[d+a \sin \frac{2 \pi}{\lambda}(x-c t)\right] .
$$

The equations for the considered flow configuration are:

$$
\begin{aligned}
& \frac{\partial u}{\partial x}+\frac{\partial v}{\partial y}=0 \\
& \rho_{e f f}\left(\frac{\partial}{\partial t}+u \frac{\partial}{\partial x}+v \frac{\partial}{\partial y}\right) u=-\frac{\partial p}{\partial x}+\mu_{e f f}\left[\frac{\partial^{2} u}{\partial x^{2}}+\frac{\partial^{2} u}{\partial y^{2}}\right] \\
& \quad-\sigma_{e f f} B_{o}^{2} u+g(\rho \beta)_{e f f}\left(T-T_{0}\right)
\end{aligned}
$$

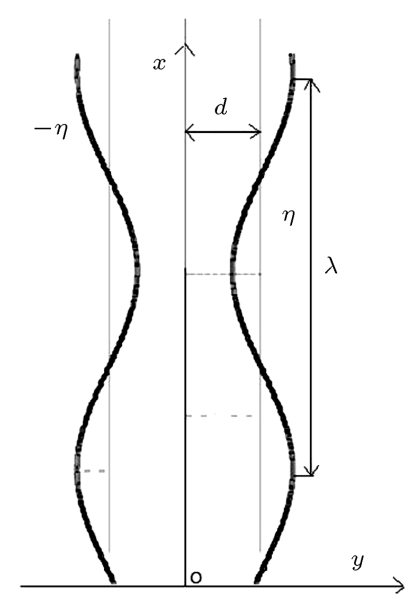

Figure 1. Flow geometry. 


$$
\begin{gathered}
\rho_{\text {eff }}\left(\frac{\partial}{\partial t}+u \frac{\partial}{\partial x}+v \frac{\partial}{\partial y}\right) v=-\frac{\partial p}{\partial y}-\sigma_{\text {eff }} B_{o}^{2} v \\
+\mu_{\text {eff }}\left[\frac{\partial^{2} v}{\partial x^{2}}+\frac{\partial^{2} v}{\partial y^{2}}\right] \\
(\rho C)_{e f f}\left(\frac{\partial}{\partial t}+u \frac{\partial}{\partial x}+v \frac{\partial}{\partial y}\right) T=\mu_{e f f}\left[\left(\frac{\partial u}{\partial y}+\frac{\partial v}{\partial x}\right)^{2}\right. \\
\left.+2\left(\left(\frac{\partial u}{\partial x}\right)^{2}+\left(\frac{\partial v}{\partial y}\right)^{2}\right)\right]+K_{\text {eff }}\left[\frac{\partial^{2} T}{\partial x^{2}}+\frac{\partial^{2} T}{\partial y^{2}}\right]
\end{gathered}
$$

The quantities assumed in the above-mentioned equations are defined as: $u$ and $v$ for components of velocity in the $x$ and $y$ directions, respectively; $\rho_{\text {eff }}$ effective density; $p$ pressure; $\mu_{\text {eff }}$ effective viscosity; $g$ acceleration due to gravity; $\sigma_{\text {eff }}$ effective thermal conductivity; and $(\rho \beta)_{e f f}, K_{\text {eff }}$, and $(\rho C)_{e f f}$ effective thermal expansion, effective thermal conductivity of nanofluids, and effective heat capacity, respectively. In addition, $T$ stands for temperature and $t$ represents time.

The notations $\rho_{\text {eff }},(\rho \beta)_{\text {eff }},(\rho C)_{\text {eff }}, \mu_{e f f}, \sigma_{\text {eff }}$, and $K_{\text {eff }}$ for the two-phase models are:

$$
\begin{aligned}
& (\rho \beta)_{e f f}=(1-\phi) \rho_{f} \beta_{f}+\phi \rho_{p} \beta_{p}, \\
& \rho_{e f f}=(1-\phi) \rho_{f}+\phi \rho_{p}, \\
& (\rho C)_{e f f}=(1-\phi)(\rho C)_{f}+\phi(\rho C)_{p}, \\
& \mu_{e f f}=\frac{\mu_{f}}{(1-\phi)^{2.5}}, \\
& \frac{\sigma_{e f f}}{\sigma_{f}}=1+\frac{3\left(\frac{\sigma_{p}}{\sigma_{f}}-1\right) \phi}{\left(\frac{\sigma_{p}}{\sigma_{f}}+2\right)-\left(\frac{\sigma_{p}}{\sigma_{f}}-1\right) \phi} .
\end{aligned}
$$

For Maxwell's model:

$$
\frac{K_{e f f}}{K_{f}}=\frac{K_{p}+2 K_{f}-2 \phi\left(K_{f}-K_{p}\right)}{K_{p}+2 K_{f}+\phi\left(K_{f}-K_{p}\right)},
$$

For Hamilton-Crosser's model:

$$
\frac{K_{e f f}}{K_{f}}=\frac{K_{p}+(n-1) K_{f}-(n-1) \phi\left(K_{f}-K_{p}\right)}{K_{p}+(n-1) K_{f}+\phi\left(K_{f}-K_{p}\right)},
$$

where the subscripts $f$ and $p$ represent the fluid and nanoparticles, and $\phi$ depicts volume fraction of nanoparticles. Two models of effective thermal conductivity are used in the above equation. The Hamilton-Crosser model is employed for the cylindrical particles with $n=6$ and Maxwell model is adopted for spherical particles. Here, $n$ represents the shape of the nanoparticles. It is defined by $3 / \Psi$, where $\Psi$ depicts sphericity of nanomaterials. The amount of $\Psi=0.5$ is used for cylindrical-shape material and $\Psi=1$ for spherical-shape material.

Thermophysical properties of the base liquid and nanoparticles are mentioned in Table 1. Dimensionless parameters are:

$$
\begin{aligned}
& x^{*}=\frac{x}{\lambda}, \quad u^{*}=\frac{u}{c}, \quad y^{*}=\frac{y}{d}, \\
& \eta^{*}=\frac{\eta}{d}, \quad v^{*}=\frac{v}{c}, \quad t^{*}=\frac{c t}{\lambda}, \\
& p *=\frac{d^{2} p}{c \lambda \mu_{f}}, \quad \theta=\frac{T-T_{0}}{T_{0}}, \quad \operatorname{Pr}=\frac{\mu_{f} C_{f}}{K_{f}}, \\
& \operatorname{Re}=\frac{\rho_{f} c d}{\mu_{f}}, \quad \operatorname{Ec}=\frac{c^{2}}{C_{f} T_{0}}, \quad \operatorname{Br}=\operatorname{Pr} E c, \\
& \mathrm{M}=\sqrt{\frac{\sigma_{f}}{\mu_{f}}} B_{o} d, \quad \operatorname{Gr}=\frac{g \rho_{f} \beta_{f} T_{0} d^{2}}{c \mu_{f}}, \\
& u=\frac{\partial \psi}{\partial y}, \quad v=-\delta \frac{\partial \psi}{\partial x} .
\end{aligned}
$$

Here, Pr, Re, Br, Ec, M, and Gr denote the Prandtl, Reynolds, Brinkman, Eckert, Hartman, and Grashof numbers, respectively.

After applying the lubrication approach, we obtained:

$$
\begin{aligned}
& \frac{\partial p}{\partial x}=\frac{1}{(1-\phi)^{2.5}} \frac{\partial^{3} \psi}{\partial y^{3}}+\mathrm{Gr} A_{3} \theta-\mathrm{M}^{2} A_{1} \frac{\partial \psi}{\partial y} \\
& \frac{\partial p}{\partial y}=0 \\
& K_{1} \frac{\partial^{2} \theta}{\partial y^{2}}+\frac{\mathrm{Br}}{(1-\phi)^{2.5}}\left(\frac{\partial^{2} \psi}{\partial y^{2}}\right)^{2}=0 \\
& A_{1}=1+\frac{3\left(\frac{\sigma_{p}}{\sigma_{f}}-1\right) \phi}{\left(\frac{\sigma_{p}}{\sigma_{f}}+2\right)-\left(\frac{\sigma_{p}}{\sigma_{f}}-1\right) \phi}
\end{aligned}
$$

Table 1. Thermophysical parameters of water and nanoparticles [13].

\begin{tabular}{cccccc}
\hline & $\begin{array}{c}\boldsymbol{\rho} \\
\left(\mathbf{k g ~ m}^{-\mathbf{3}}\right)\end{array}$ & $\begin{array}{c}\boldsymbol{C}_{\boldsymbol{p}} \\
\left(\mathbf{j ~ k g}^{-\mathbf{1}} \mathbf{K}^{-\mathbf{1}}\right)\end{array}$ & $\begin{array}{c}\boldsymbol{K} \\
\left(\mathbf{W ~ m}^{-\mathbf{1}} \mathbf{K}^{-\mathbf{1}}\right)\end{array}$ & $\begin{array}{c}\boldsymbol{\beta} \\
(\mathbf{l} / \mathbf{k}) \times \mathbf{1 0}^{-\mathbf{6}}\end{array}$ & $\begin{array}{c}\boldsymbol{\sigma} \\
(\boldsymbol{\Omega . m})^{-\mathbf{1}}\end{array}$ \\
\hline $\mathbf{F e}_{\mathbf{3}} \mathbf{O}_{\mathbf{4}}$ & 5200 & 670 & 80.6 & 13 & 25000 \\
$\mathbf{H}_{\mathbf{2}} \mathbf{O}$ & 997.1 & 4179 & 0.613 & 210 & 0.05 \\
\hline
\end{tabular}




$$
A_{3}=1-\phi+\phi\left(\frac{(\rho \beta)_{p}}{(\rho \beta)_{f}}\right)
$$

For Maxwell's model:

$$
K_{1}=\frac{K_{p}+2 K_{f}-2 \phi\left(K_{f}-K_{p}\right)}{K_{p}+2 K_{f}+\phi\left(K_{f}-K_{p}\right)},
$$

For Hamilton-Crosser's model:

$$
K_{1}=\frac{K_{p}+(n-1) K_{f}-(n-1) \phi\left(K_{f}-K_{p}\right)}{K_{p}+(n-1) K_{f}+\phi\left(K_{f}-K_{p}\right)} .
$$

Boundary conditions become:

$$
\begin{aligned}
& \frac{\partial \psi}{\partial y} \pm \beta_{1} \frac{\partial^{2} \psi}{\partial y^{2}} \pm \beta_{2} \frac{\partial^{3} \psi}{\partial y^{3}}=0, \\
& \theta \pm \gamma_{1} \frac{\partial \theta}{\partial y} \pm \gamma_{2} \frac{\partial^{2} \theta}{\partial y^{2}}=0, \quad \text { at } y= \pm \eta \\
& {\left[E_{1} \frac{\partial^{3}}{\partial x^{3}}+E_{2} \frac{\partial^{3}}{\partial x \partial t^{2}}+E_{3} \frac{\partial^{2}}{\partial t \partial x}\right] \eta=\frac{1}{(1-\phi)^{2.5}} \frac{\partial^{3} \psi}{\partial y^{3}}} \\
& +\operatorname{Gr} A_{3} \theta-\mathrm{M}^{2} A_{1} \frac{\partial \psi}{\partial y}, \quad \text { at } y= \pm \eta .
\end{aligned}
$$

Here, velocity and temperature slip parameters in dimensionless form are denoted by $\beta_{1}, \beta_{2}$ and $\gamma_{1}, \gamma_{2}$, respectively.

\subsection{Entropy analysis}

Viscous dissipation is represented by:

$$
\Phi=\mu_{e f f}\left[\left(\frac{\partial u}{\partial y}+\frac{\partial v}{\partial x}\right)^{2}+2\left(\left(\frac{\partial u}{\partial x}\right)^{2}+\left(\frac{\partial v}{\partial y}\right)^{2}\right)\right]
$$

Volumetric entropy generation in dimensional form is:

$$
S_{g e n}^{\prime \prime \prime}=\frac{K_{e f f}}{T_{m}^{2}}\left(\left(\frac{\partial T}{\partial x}\right)^{2}+\left(\frac{\partial T}{\partial y}\right)^{2}\right)+\frac{\Phi}{T_{m}} .
$$

Dimensionless form becomes:

$$
\begin{aligned}
& N_{s}=\frac{S_{g e n}^{\prime \prime \prime}}{S_{G}^{\prime \prime \prime}}=K_{1}\left(\frac{\partial \theta}{\partial y}\right)^{2}+\frac{\mathrm{Br}}{\Lambda(1-\phi)^{2.5}}\left(\frac{\partial^{2} \psi}{\partial y^{2}}\right)^{2}, \\
& S_{G}^{\prime \prime \prime}=\frac{K_{f} T_{0}^{2}}{T_{m}^{2} d^{2}}, \quad \Lambda=\frac{T_{0}}{T_{m}} .
\end{aligned}
$$

Bejan number is:

$$
\mathrm{Be}=\frac{N s_{\text {cond }}}{N s_{\text {cond }}+N s_{v i s c}} .
$$

Eq. (15) comprises two parts; $\left(N s_{\text {cond }}\right)$ is entropy generation due to temperature difference and $N s_{v i s c}$ is entropy generation due to viscous dissipation.

\section{Solution methodology}

Perturbation technique is employed to achieve the solution. The small Grashof number is chosen as the perturbation parameter and the corresponding systems and their solutions are:

Zeroth-order systems. Here we have:

$$
\begin{aligned}
& \frac{1}{(1-\phi)^{2.5}} \frac{\partial^{4} \psi_{0}}{\partial y^{4}}-M^{2} A_{1} \frac{\partial^{2} \psi_{0}}{\partial y^{2}}=0 \\
& K_{1} \frac{\partial^{2} \theta_{0}}{\partial y^{2}}+\frac{\mathrm{Br}}{(1-\phi)^{2.5}}\left(\frac{\partial^{2} \psi_{0}}{\partial y^{2}}\right)^{2}=0 \\
& \frac{\partial \psi_{0}}{\partial y} \pm \beta_{1} \frac{\partial^{2} \psi_{0}}{\partial y^{2}} \pm \beta_{2} \frac{\partial^{3} \psi_{0}}{\partial y^{3}}=0, \quad \text { at } y= \pm \eta \\
& {\left[E_{1} \frac{\partial^{3}}{\partial x^{3}}+E_{2} \frac{\partial^{3}}{\partial x \partial t^{2}}+E_{3} \frac{\partial^{2}}{\partial t \partial x}\right] \eta=\frac{1}{(1-\phi)^{2.5}} \frac{\partial^{3} \psi_{0}}{\partial y^{3}}} \\
& -M^{2} A_{1} \frac{\partial \psi_{0}}{\partial y}, \quad \text { at } y= \pm \eta \\
& \theta_{0} \pm \gamma_{1} \frac{\partial \theta_{0}}{\partial y} \pm \gamma_{2} \frac{\partial^{2} \theta_{0}}{\partial y^{2}}=0, \quad \text { at } y= \pm \eta
\end{aligned}
$$

The corresponding stream function and temperature solutions are:

$$
\psi_{0}=\frac{A_{0} e^{\frac{-\sqrt{A_{1}} M y}{\sqrt{A_{0}}}}\left(e^{\frac{2 \sqrt{A_{1}} M y}{\sqrt{A_{0}}}} C_{1}+C_{2}\right)}{A_{1} M^{2}}+C_{3}+y C_{4},
$$

$$
\begin{aligned}
\theta_{0}= & -\frac{1}{4 A_{1} K_{1} M^{2}} A_{0} \operatorname{Br}\left(e ^ { \frac { - 2 \sqrt { A _ { 1 } } M y } { \sqrt { A _ { 0 } } } } \left(C_{2}^{2}\right.\right. \\
& \left.\left.+C_{1}^{2} e^{\frac{4 \sqrt{A_{1}} M y}{\sqrt{A_{0}}}}\right)\right)+F_{1}+y F_{2} .
\end{aligned}
$$

First-order systems. Here we have:

$$
\begin{aligned}
& \frac{1}{(1-\phi)^{2.5}} \frac{\partial^{4} \psi_{1}}{\partial y^{4}}+A_{3} \frac{\partial \theta_{0}}{\partial y}-M^{2} A_{1} \frac{\partial^{2} \psi_{1}}{\partial y^{2}}=0 \\
& K_{1} \frac{\partial^{2} \theta_{1}}{\partial y^{2}}+\frac{B r}{(1-\phi)^{2.5}}\left(2 \frac{\partial^{2} \psi_{0}}{\partial y^{2}} \frac{\partial^{2} \psi_{1}}{\partial y^{2}}\right)=0 \\
& \frac{\partial \psi_{1}}{\partial y} \pm \beta_{1} \frac{\partial^{2} \psi_{1}}{\partial y^{2}} \pm \beta_{2} \frac{\partial^{3} \psi_{1}}{\partial y^{3}}=0, \quad \text { at } \quad y= \pm \eta \\
& \frac{1}{(1-\phi)^{2.5}} \frac{\partial^{3} \psi_{1}}{\partial y^{3}}+A_{3} \theta_{0}-M^{2} A_{1} \frac{\partial \psi_{1}}{\partial y}=0 \\
& \text { at } y= \pm \eta, \quad \text { at } y= \pm \eta \\
& \theta_{1} \pm \gamma_{1} \frac{\partial \theta_{1}}{\partial y} \pm \gamma_{2} \frac{\partial^{2} \theta_{1}}{\partial y^{2}}=0, \quad y
\end{aligned}
$$

The solution expressions are: 


$$
\begin{aligned}
& \psi_{1}=-\frac{1}{24 A_{1}^{2} K_{1} M^{5}} \\
& \left(\frac{A_{0}^{\frac{5}{2}} \frac{9}{8} A_{3} B r e^{\frac{-\sqrt{A_{1}} M y}{\sqrt{A_{0}}}}\left(C_{2}^{2}-C_{1}^{2} e^{\frac{4 \sqrt{A_{1}} M y}{\sqrt{A_{0}}}}\right)}{\sqrt{A_{1}}}\right. \\
& -12 A_{1} A_{3} F_{2} K_{1} M^{3} y^{2}+8 A_{0} A_{1} M^{3}\left(A_{3} B r C_{1} C_{2} y^{3}\right. \\
& \left.-3 e^{\frac{-\sqrt{A_{1}} M y}{\sqrt{A_{0}}}} K_{1}\left(e^{\frac{2 \sqrt{A_{1}} M y}{\sqrt{A_{0}}}} B_{1}+B_{2}\right)\right) \\
& +y B_{4}+B_{3},
\end{aligned}
$$

$\theta_{1}=\frac{1}{9 A_{1}^{2} K_{1} M^{5}} A_{0} B r\left(\frac{A_{0}^{\frac{5}{2}} A_{3} B r C_{2}^{3} e^{\frac{-3 \sqrt{A_{1}} M y}{\sqrt{A_{0}}}}}{3 \sqrt{A_{1}} M}\right.$

$$
\begin{aligned}
& -\frac{A_{0}^{\frac{5}{2}} A_{3} B r C_{1}^{3} e^{\frac{3 \sqrt{A_{1}} M y}{\sqrt{A_{0}}}}}{3 \sqrt{A_{1}} M} \\
& -\frac{9}{2} A_{0} A_{1} B_{2} C_{2} e^{\frac{-2 \sqrt{A_{1}} M y}{\sqrt{A_{0}}}} K_{1} M^{2} \\
& -\frac{9}{2} A_{0} A_{1} B_{1} C_{1} e^{\frac{2 \sqrt{A_{1}} M y}{\sqrt{A_{0}}}} K_{1} M^{2} \\
& -9 A_{1}^{2}\left(B_{2} C_{1}+B_{1} C_{2}\right) K_{1} M^{4} y^{2} \\
& -3 \sqrt{A_{0}} A_{3} C_{2} e^{\frac{-\sqrt{A_{1}} M y}{\sqrt{A_{0}}}} \\
& \left(\frac{\sqrt{A_{0}}\left(-25 A_{0}^{\frac{3}{2}} B r C_{1} C_{2}+6 \sqrt{A_{1}} F_{2} K_{1} M\right)}{\sqrt{A_{1}} M}\right. \\
& \left.\left.-12 A_{0}^{\frac{3}{2}} B r C_{1} C_{2} y\right)-3 \sqrt{A_{0}} A_{3} C_{1} e^{\frac{\sqrt{A_{1}} M y}{\sqrt{A_{0}}}}\right) \\
& \left(\frac{\sqrt{A_{0}}\left(25 A_{0}^{\frac{3}{2}} B r C_{1} C_{2}+6 \sqrt{A_{1}} F_{2} K_{1} M\right)}{\sqrt{A_{1}} M}\right. \\
& \left.-12 A_{0}^{\frac{3}{2}} B r C_{1} C_{2} y\right)+G_{1}+y G_{2} .
\end{aligned}
$$

The constants $B i$ 's, $C i$ 's, Gi's, and Fi's can be computed via Mathematica.

\section{Discussion}

This section is devoted to the analysis of velocity, temperature, entropy generation, Bejan number, and stream lines. Each quantity is analyzed in a specific subsection.

\subsection{Analysis of velocity}

In this subsection, behavior of velocity is discussed under the influence of different important parameters. Figure 2 represents the impact of nanoparticle volume fraction on velocity profile. This graph shows decreasing behavior, which is due to the fact that by increasing the quantity of nanoparticles $(\phi=$ $0.01,0.03,0.05,0.07)$, resistance to the fluid increases so that fluid velocity diminishes. In this study, the values for the Hamilton-Crosser's model are greater than those for the Maxwell's model. Figure 3 presents velocity against Hartman number. It demonstrates that velocity has decreasing behavior with increase in Hartman number $(\mathrm{M}=2,3,4,5)$, because the Lorentz

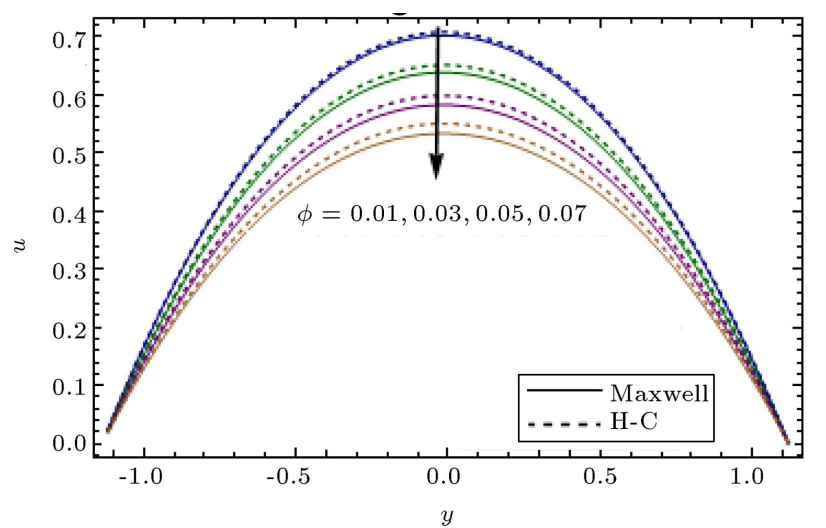

Figure 2. $u$ in terms of $\phi$ when $t=0.1, E_{3}=0.01$, $x=0.2, E_{2}=0.01, E_{1}=0.02, M=1.0, \varepsilon=0.2$, $\mathrm{Gr}=0.03, \mathrm{Br}=3.0, \beta_{1}=0.01, \beta_{2}=-0.01, \gamma_{1}=0.01$, and $\gamma_{2}=-0.01$.

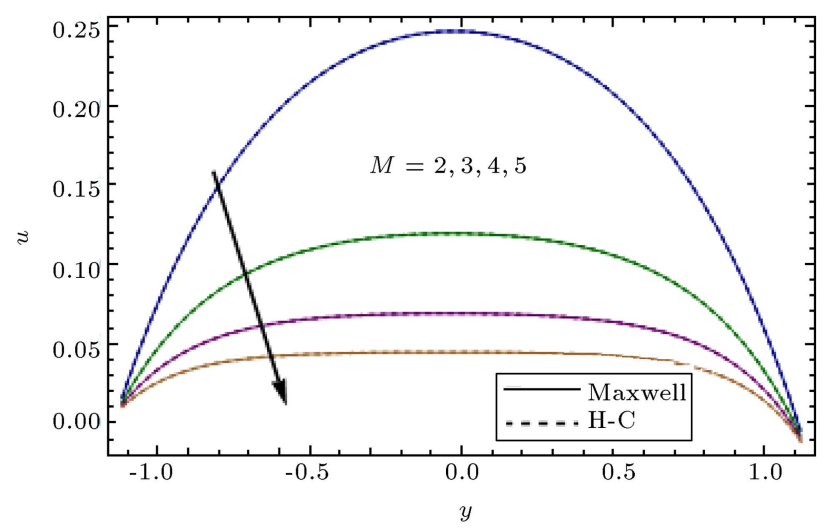

Figure 3. $u$ in terms of $M$ when $t=0.1, E_{3}=0.01$, $x=0.2, E_{2}=0.01, E_{1}=0.02, \varepsilon=0.2, \phi=0.01, \mathrm{Br}=3.0$, $\mathrm{Gr}=0.03, \beta_{1}=0.01, \beta_{2}=-0.01, \gamma_{1}=0.01$, and $\gamma_{2}=-0.01$. 


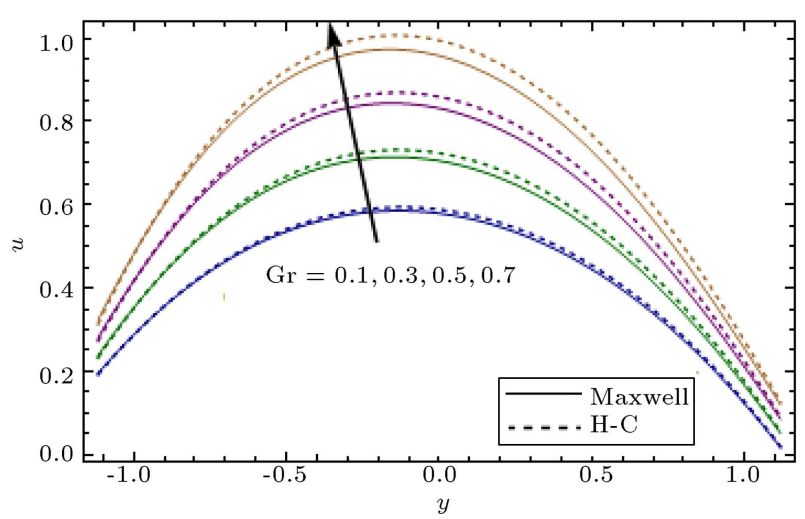

Figure 4. $u$ in terms of Gr when $t=0.1, E_{3}=0.01$, $x=0.2, E_{2}=0.01, E_{1}=0.02, \varepsilon=0.2, \mathrm{M}=1.0, \phi=0.1$, $\mathrm{Br}=3.0, \beta_{1}=0.01, \beta_{2}=-0.01, \gamma_{1}=0.01$, and $\gamma_{2}=-0.01$.

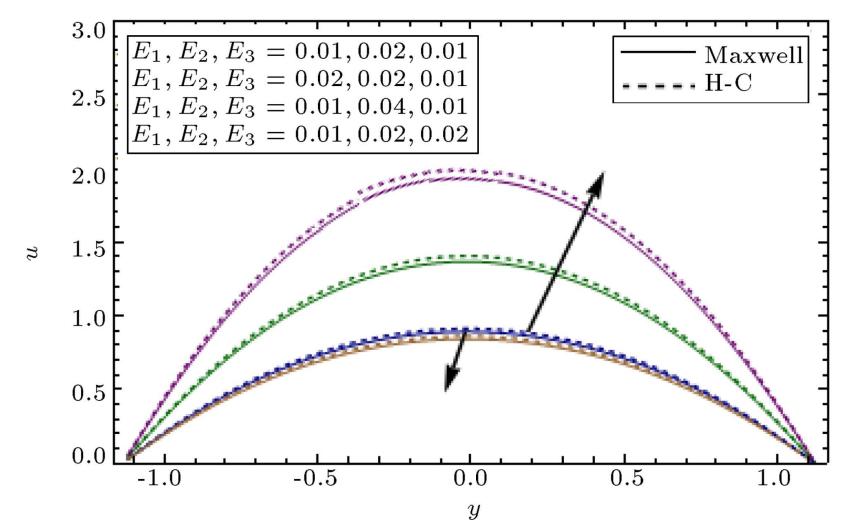

Figure 5. $u$ in terms of $E_{1}, E_{2}, E_{3}$ when $t=0.1, x=0.2$, $\varepsilon=0.2, \mathrm{M}=1.0, \phi=0.1, \mathrm{Br}=3.0, \mathrm{Gr}=0.03, \beta_{1}=0.01$, $\beta_{2}=-0.01, \gamma_{1}=0.01$, and $\gamma_{2}=-0.01$.

force acts as a resistive force. Figure 4 deals with Grashof number, showing increase in velocity profile by enhancing Grashof number $(\mathrm{Gr}=0.1-0.7)$. This is due to increase in buoyancy forces, which facilitate the flow. Velocity profile with wall parameters can be observed in Figure 5. The results illustrate that velocity increases with elastance parameters $\left(E_{1}=0.01,0.02\right)$ and $\left(E_{2}=\right.$ $0.02,0.04)$ and decreases with the damping parameter $\left(E_{3}=0.01,0.02\right)$. Obviously, elastance parameters cause lower resistance, leading to increase in velocity, whereas damping resists the flow. The results for the slip parameters are demonstrated in Figures 6 and 7 . As shown, velocity profile shows enhancement when the slip parameters $\left(\beta_{1}=0.1,0.3,0.5,0.7\right)$ and $\left(\beta_{2}=-0.1,-0.3,-0.5,-0.7\right)$ are increased. It is also noteworthy that this behavior is more prominent with the second-order slip parameter than with the first-order one. Furthermore, the velocity profile is higher in Hamilton-Crosser's model than in Maxwell's model.

\subsection{Analysis of temperature}

In this subsection, temperature profile for different per-

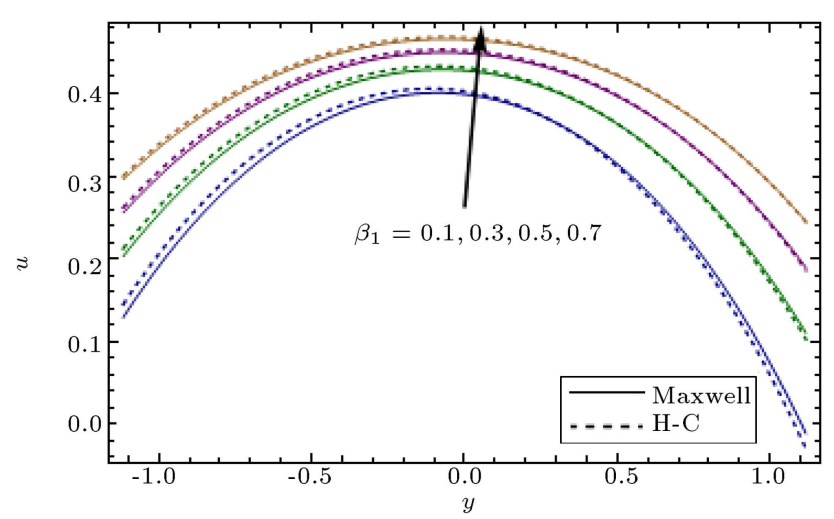

Figure 6. $u$ in terms of $\beta_{1}$ when $t=0.1, E_{3}=0.01$, $x=0.2, E_{2}=0.01, E_{1}=0.02, \varepsilon=0.2, \mathrm{M}=1.0, \phi=0.1$, $\mathrm{Br}=3.0, \mathrm{Gr}=0.03, \beta_{2}=-0.01, \gamma_{1}=0.01$, and $\gamma_{2}=-0.01$.

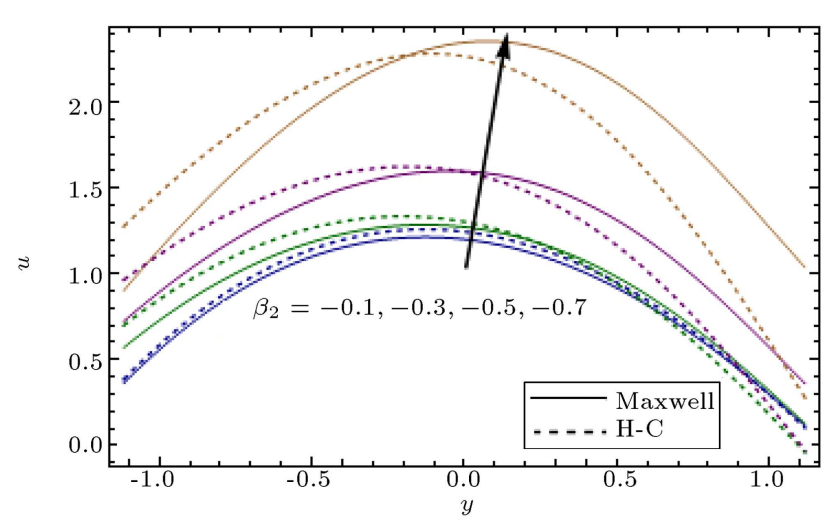

Figure 7. $u$ in terms of $\beta_{2}$ when $t=0.1, E_{3}=0.01$, $x=0.2, E_{2}=0.01, E_{1}=0.02, \varepsilon=0.2, \mathrm{M}=1.0, \phi=0.1$, $\mathrm{Br}=3.0, \mathrm{Gr}=0.03, \beta_{1}=0.01, \gamma_{1}=0.01$, and $\gamma_{2}=-0.01$.

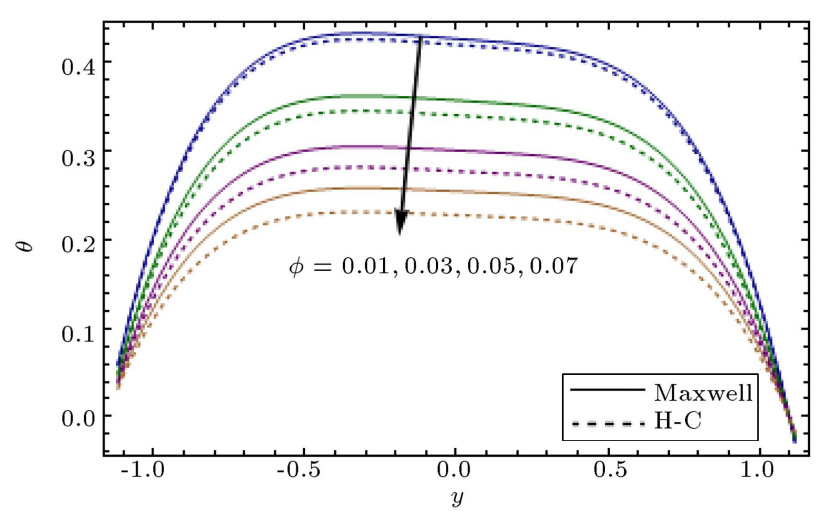

Figure 8. $\theta$ in terms of $\phi$ when $t=0.1, E_{3}=0.01$, $x=0.2, E_{2}=0.01, E_{1}=0.02, \varepsilon=0.2, \mathrm{M}=1.0$, $\mathrm{Gr}=0.03, \mathrm{Br}=3.0, \beta_{1}=0.01, \beta_{2}=-0.01, \gamma_{1}=0.01$, and $\gamma_{2}=-0.01$.

tinent parameters is accounted for. Figure 8 provides graphs for $\phi=0.01,0.03,0.05$, and 0.07 versus temperature distribution. This graph illustrates that temperature profile diminishes with increasing $\phi$. On the other hand, increase in $\phi$ enhances thermal conductivity and 


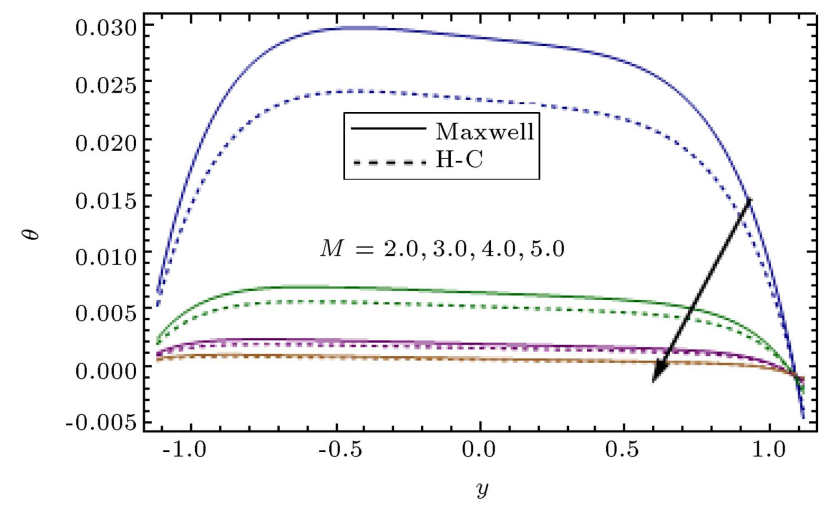

Figure 9. $\theta$ in terms of $M$ when $t=0.1, E_{3}=0.01$, $x=0.2, E_{2}=0.01, E_{1}=0.02, \varepsilon=0.2, \phi=0.1, \mathrm{Br}=3.0$, $\mathrm{Gr}=0.03, \beta_{1}=0.01, \beta_{2}=-0.01, \gamma_{1}=0.01$, and $\gamma_{2}=-0.01$.

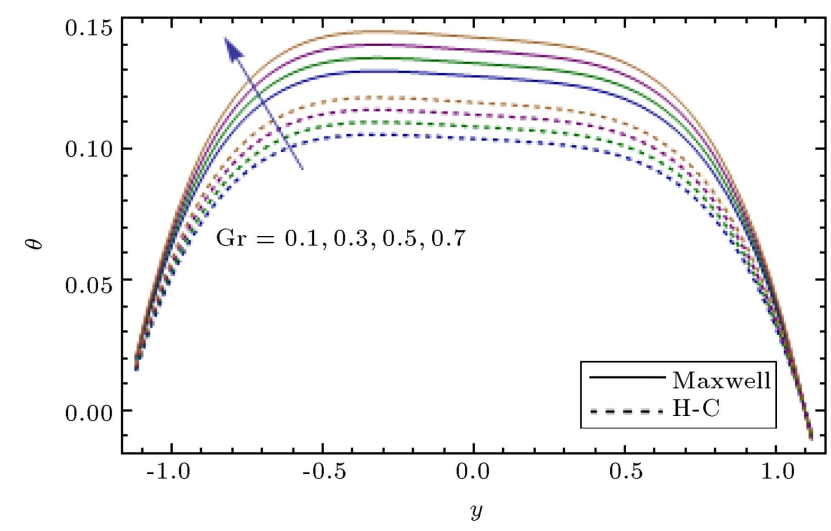

Figure 10. $\theta$ in terms of $\mathrm{Gr}$ when $t=0.1, E_{3}=0.01$, $x=0.2, E_{2}=0.01, E_{1}=0.02, \varepsilon=0.2, \mathrm{M}=1.0, \phi=0.1$, $\mathrm{Br}=3.0, \beta_{1}=0.01, \beta_{2}=-0.01, \gamma_{1}=0.01$, and $\gamma_{2}=-0.01$.

cooling capabilities. Moreover, temperature is higher in Maxwell's model than in the Hamilton-Crosser's model. Figure 9 plots the results for Hartman number versus $\theta$, demonstrating the decreasing behavior of temperature when Hartman number increases from 2 to 5 . Figure 10 shows that temperature profile increases with Grashof number $(0.1-0.7)$. Gr increases the velocity, in turn leading to increase also in the mean kinetic energy of particles as well as in temperature. The impact of wall parameters is elucidated in Figure 11. The figure shows the same behavior as in the case of velocity profile with the variation of parameters $\left(E_{1}=0.01,0.02\right)$, $\left(E_{2}=0.02,0.04\right)$, and $\left(E_{3}=0.01,0.02\right)$. The reasons can be related to velocity. The results for the first- and second-order thermal slip parameters can be observed in Figures 12 and 13, respectively. For the first-order thermal slip, the values of $\left(\gamma_{1}=0.01,0.03,0.05,0.07\right)$ lead to increase in temperature throughout the channel whereas for the second-order thermal slip, in the range of $\left(\gamma_{2}=-0.01\right.$ to -0.07$)$, temperature increases near the center. A comparative observation reveals

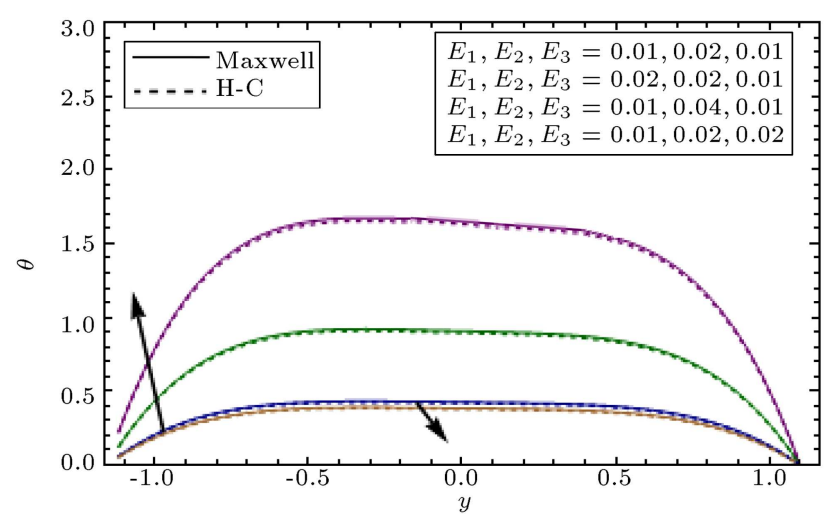

Figure 11. $\theta$ in terms of $E_{1}, E_{2}, E_{3}$ when $t=0.1$, $x=0.2, \varepsilon=0.2, \mathrm{M}=1.0, \phi=0.1, \mathrm{Br}=3.0, \mathrm{Gr}=0.03$, $\beta_{1}=0.01, \beta_{2}=-0.01, \gamma_{1}=0.01$, and $\gamma_{2}=-0.01$.

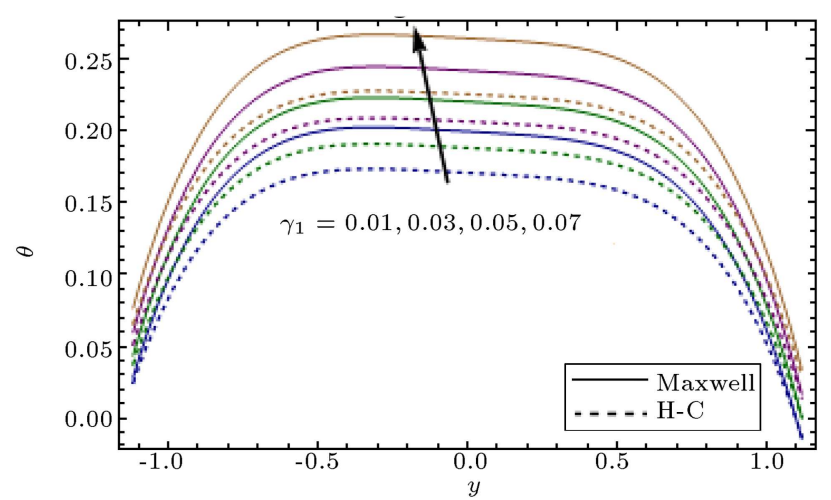

Figure 12. $\theta$ in terms of $\gamma_{1}$ when $t=0.1, E_{3}=0.01$, $x=0.2, E_{2}=0.01, E_{1}=0.02, \varepsilon=0.2, \mathrm{M}=1.0, \phi=0.1$, $\mathrm{Br}=3.0, \mathrm{Gr}=0.03, \beta_{1}=0.01, \beta_{2}=-0.01$, and $\gamma_{2}=-0.01$.

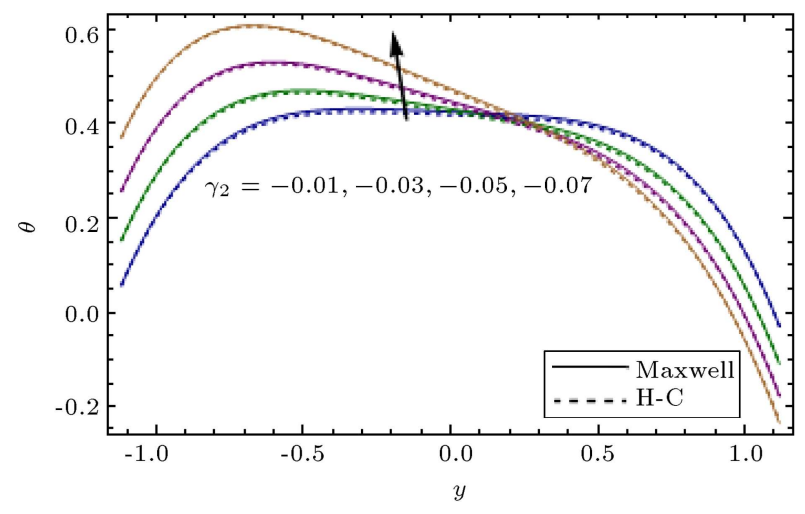

Figure 13. $\theta$ in terms of $\gamma_{2}$ when $t=0.1, E_{3}=0.01$, $x=0.2, E_{2}=0.01, E_{1}=0.02, \varepsilon=0.2, \mathrm{M}=1.0, \phi=0.1$, $\mathrm{Br}=3.0, \mathrm{Gr}=0.03, \beta_{1}=0.01, \beta_{2}=-0.01$, and $\gamma_{1}=0.01$.

that temperature remains higher for spherical-shape particles than for cylindrical-shape ones.

\subsection{Analysis of entropy generation and Bejan number}

This subsection accounts for entropy generation and Bejan number for different embedded parameters. Fig- 


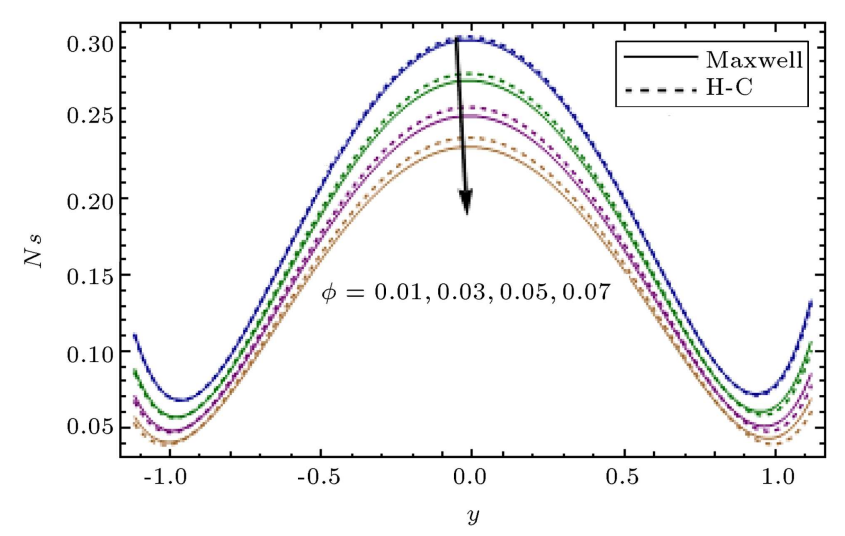

Figure 14. $N s$ in terms of $\phi$ when $t=0.1, E_{3}=0.01$, $x=0.2, E_{2}=0.01, E_{1}=0.02, \varepsilon=0.2, \mathrm{M}=1.0$, $\operatorname{Br} \Lambda^{-1}=1.0, \mathrm{Gr}=0.03, \mathrm{Br}=3.0, \beta_{1}=0.01$, $\beta_{2}=-0.01, \gamma_{1}=0.01$, and $\gamma_{2}=-0.01$.

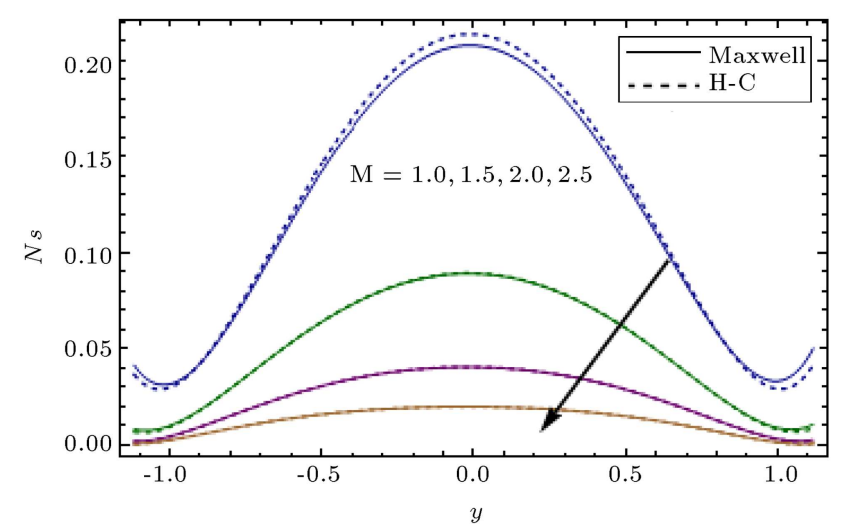

Figure 15. $N s$ in terms of $\mathrm{M}$ when $t=0.1, E_{3}=0.01$, $x=0.2, E_{2}=0.01, E_{1}=0.02, \varepsilon=0.2, \phi=0.1$,

$\mathrm{Br} \Lambda^{-1}=1.0, \mathrm{Gr}=0.03, \mathrm{Br}=3.0, \beta_{1}=0.01, \beta_{2}=-0.01$, $\gamma_{1}=0.01$, and $\gamma_{2}=-0.01$.

ure 14 illustrates the impact of $\phi$ on entropy generation. The results indicate that entropy generation decreases with larger values of $\phi$, i.e. $0.01,0.03,0.05$, and 0.07. It is due to decrease in temperature with larger volume fractions of nanoparticles considering the fact that entropy of the system is directly dependent on temperature. Figure 15 portrays the results for Hartman number. With larger values of Hartman number $(1.0,1.5,2.0$, and 2.5), entropy generation decreases. Figure 16 shows that Grashof number has increasing impact on $N s$ as $\mathrm{Gr}$ takes values between $(0.1-0.7)$. The results for this case are qualitatively similar to those for temperature. According to Figure 17 , entropy generation is enhanced when the ratio of $\mathrm{Br}$ to $\Lambda$ increases $(0.1-0.7)$. Figure 18 gives the information on the influence of wall parameters. Entropy generation is an increasing function of $E_{1}(=$ $0.01,0.02)$ and $E_{2}(=0.02,0.04)$ whereas it decreases with $E_{3}(=0.01,0.02)$. For all cases, the values in the Hamilton-Crosser's model are greater than those in the Maxwell's model.

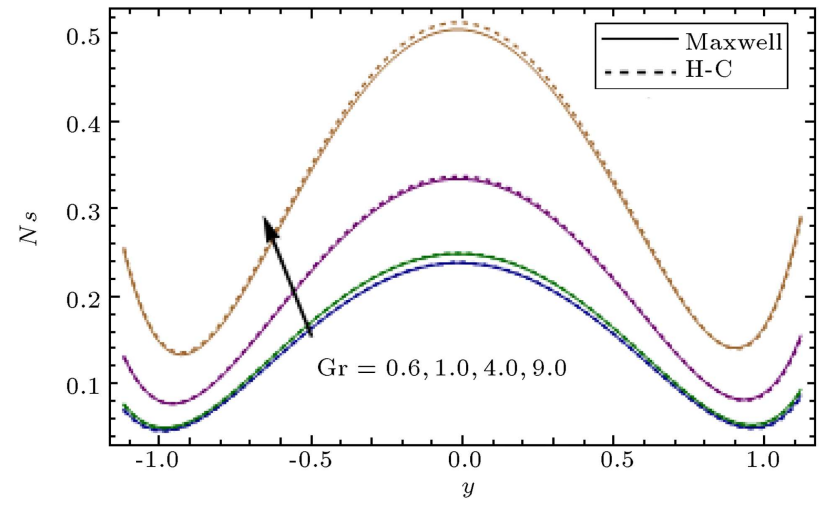

Figure 16. $N s$ in terms of $\mathrm{Gr}$ when $t=0.1, E_{3}=0.01$, $x=0.2, E_{2}=0.01, E_{1}=0.02, \varepsilon=0.2, \phi=0.1, \mathrm{M}=1.0$, $\mathrm{Br} \Lambda^{-1}=1.0, \mathrm{Br}=3.0, \beta_{1}=0.01, \beta_{2}=-0.01, \gamma_{1}=0.01$, and $\gamma_{2}=-0.01$.

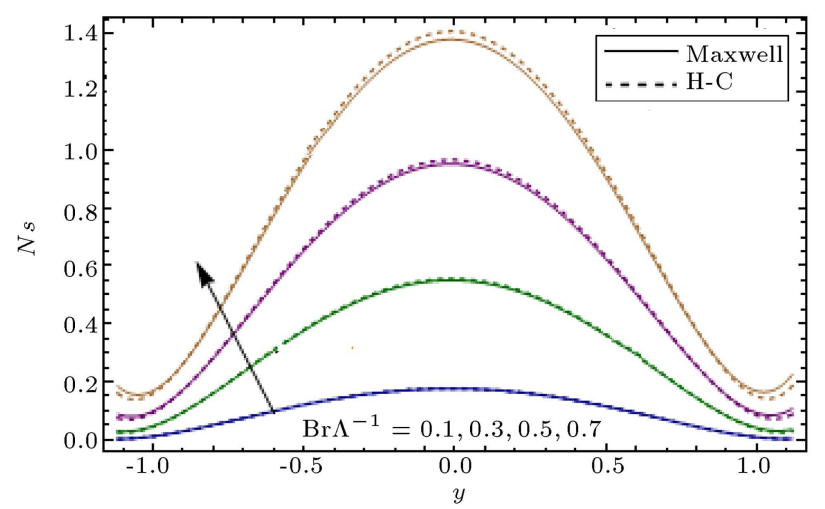

Figure 17. $N s$ in terms of $\operatorname{Br} \Lambda^{-1}$ when $t=0.1$, $E_{3}=0.01, x=0.2, E_{2}=0.01, E_{1}=0.02, \varepsilon=0.2, \phi=0.1$, $\mathrm{M}=1.0, \mathrm{Gr}=0.03, \mathrm{Br}=3.0, \beta_{1}=0.01, \beta_{2}=-0.01$, $\gamma_{1}=0.01$, and $\gamma_{2}=-0.01$.

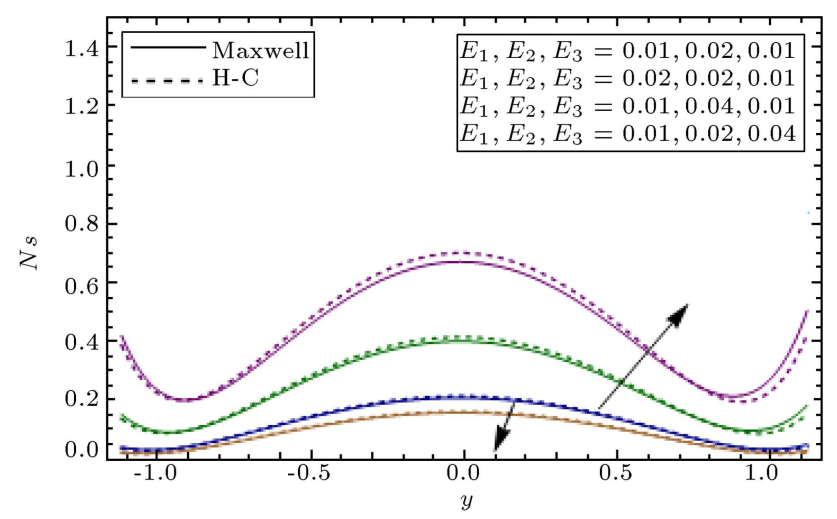

Figure 18. $N s$ in terms of $E_{1}, E_{2}$, and $E_{3}$ when $t=0.1$, $x=0.2, \varepsilon=0.2, \phi=0.1, \mathrm{M}=1.0, \mathrm{Br} \Lambda^{-1}=1.0$, $\mathrm{Gr}=0.03, \mathrm{Br}=3.0, \beta_{1}=0.01, \beta_{2}=-0.01, \gamma_{1}=0.01$, and $\gamma_{2}=-0.01$.

Figures 19-23 demonstrate the behavior of Bejan number with pertinent parameters. Figure 19 displays the impact of nanoparticles volume fraction on Be. The relation between Bejan number and nanoparticles volume fraction is inverse as increase in $\phi(0.1-0.7)$ 


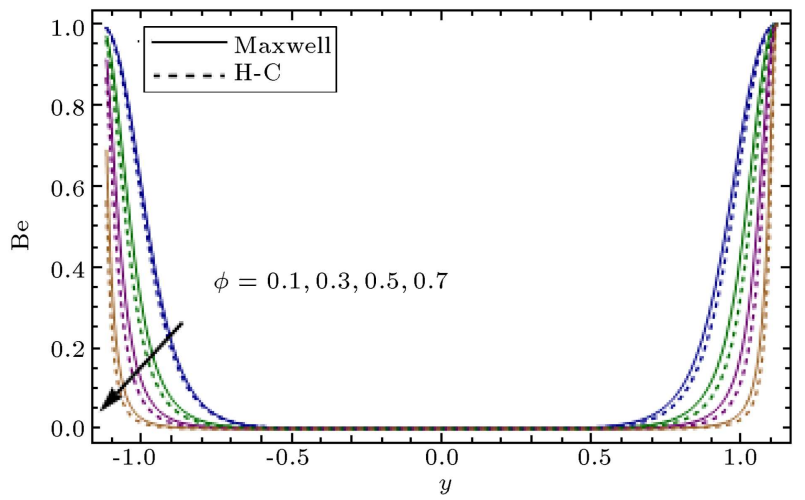

Figure 19. Be in terms of $\phi$ when $t=0.1, E_{3}=0.01$, $x=0.2, E_{2}=0.01, E_{1}=0.02, \varepsilon=0.2, \mathrm{M}=1.0$, $\mathrm{Br} \Lambda^{-1}=1.0, \mathrm{Gr}=0.03, \mathrm{Br}=3.0, \beta_{1}=0.01, \beta_{2}=-0.01$, $\gamma_{1}=0.01$, and $\gamma_{2}=-0.01$.

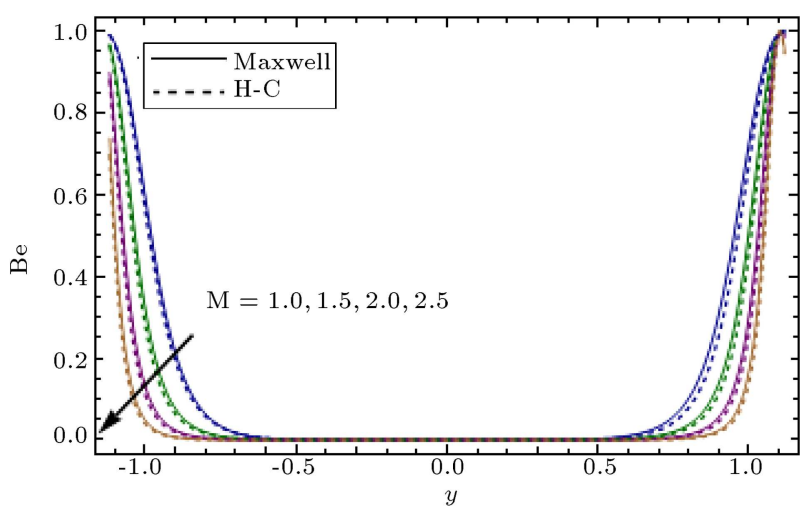

Figure 20. Be in terms of $\mathrm{M}$ when $t=0.1, E_{3}=0.01$, $x=0.2, E_{2}=0.01, E_{1}=0.02, \varepsilon=0.2, \phi=0.1$, $\mathrm{Br} \Lambda^{-1}=1.0, \mathrm{Gr}=0.03, \mathrm{Br}=3.0, \beta_{1}=0.01, \beta_{2}=-0.01$, $\gamma_{1}=0.01$, and $\gamma_{2}=-0.01$.

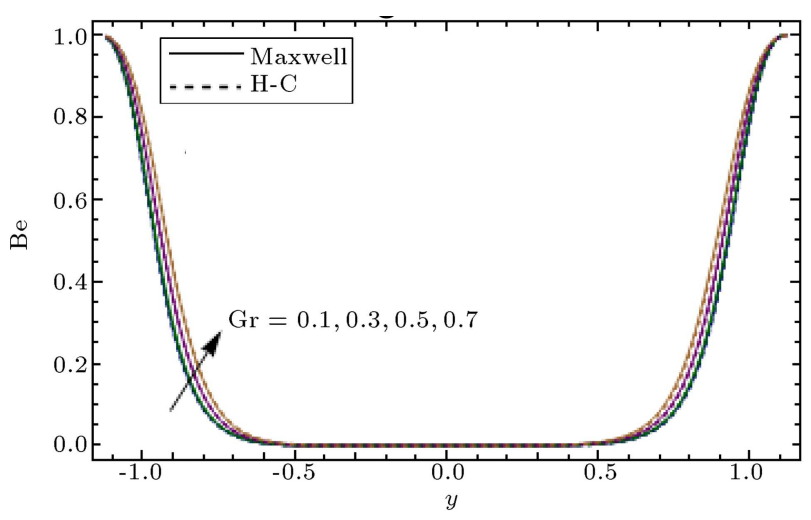

Figure 21. Be in terms of $\mathrm{Gr}$ when $t=0.1, E_{3}=0.01$, $x=0.2, E_{2}=0.01, E_{1}=0.02, \varepsilon=0.2, \phi=0.1, \mathrm{M}=1.0$, $\mathrm{Br} \Lambda^{-1}=1.0, \mathrm{Br}=3.0, \beta_{1}=0.01, \beta_{2}=-0.01, \gamma_{1}=0.01$, and $\gamma_{2}=-0.01$.

causes the decrease in Bejan number. As shown in Figure 20, increase in Hartman number from 1.0 to 2.5 decreases the Bejan number. Figure 21 indicates direct relation between Grashof number (from 0.1 to 0.7 ) and Bejan number. According to Figure 22,

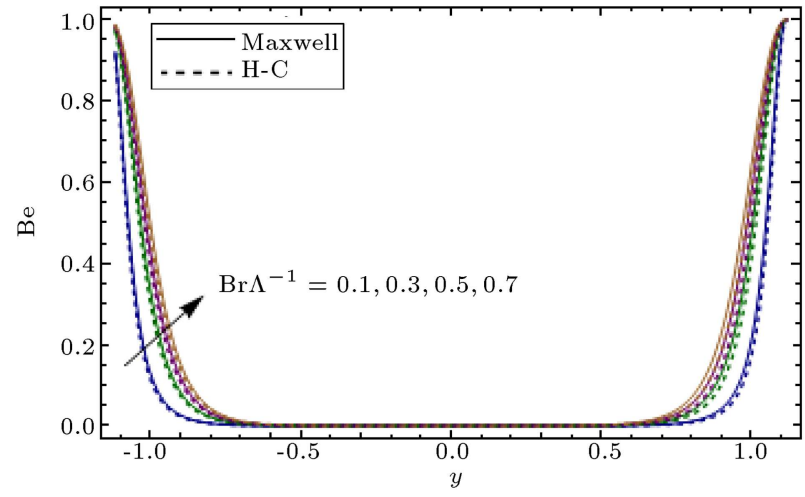

Figure 22. Be in terms of $\operatorname{Br} \Lambda^{-1}$ when $t=0.1$, $E_{3}=0.01, x=0.2, E_{2}=0.01, E_{1}=0.02, \varepsilon=0.2, \phi=0.1$, $\mathrm{M}=1.0, \mathrm{Gr}=0.03, \mathrm{Br}=3.0, \beta_{1}=0.01, \beta_{2}=-0.01$, $\gamma_{1}=0.01$, and $\gamma_{2}=-0.01$.

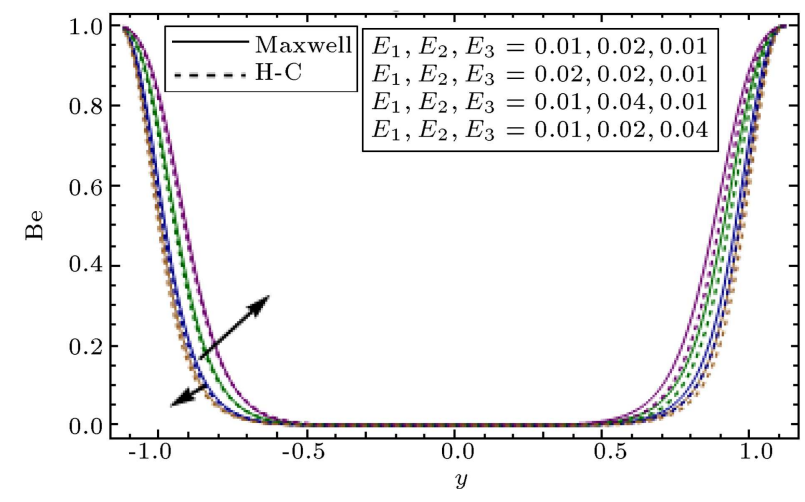

Figure 23. Be in terms of $E_{1}, E_{2}$, and $E_{3}$ when $t=0.1$, $x=0.2, \varepsilon=0.2, \phi=0.1, \mathrm{M}=1.0, \mathrm{Br} \Lambda^{-1}=1.0$,

$\mathrm{Gr}=0.03, \mathrm{Br}=3.0, \beta_{1}=0.01, \beta_{2}=-0.01, \gamma_{1}=0.01$, and $\gamma_{2}=-0.01$.

Bejan number increases with rise in ratio of $\mathrm{Br}$ to $\Lambda$ from 0.1 to 0.7. Figure 23 presents the results for wall parameters. While enhancement is observed in Bejan number with larger elastance parameters, i.e. $E_{1}(=0.01,0.02)$ and $E_{2}(=0.02,0.04)$, it is decreased with larger values of the damping parameter, i.e. $E_{3}(=$ 0.01, 0.02). Moreover, in all cases, the values of the parameters in Hamilton-Crosser's model are less than those in Maxwell's model.

\subsection{Streamlines}

The streamlines are plotted to illustrate trapping. Figure 24(a) and (b) shows the impact of Hartman number in Maxwell model and Figure 24(c) and (d) portrays it in Hamilton-Crosser model. In both cases, the size of trapped bolus increases with larger values of Hartman number $(\mathrm{M}=1.0,2.0)$. Figures 25 and 26(a)-(d) picture the behavior of the first- and secondorder slip parameters. The streamlines indicate that the size of the trapped bolus rises with enhancement in the first-order slip (from 0.01 to 0.03 ) and secondorder slip parameter $(-0.01,-0.03)$. The impact of wall parameters can be observed in Figure 27(a)-(d) 


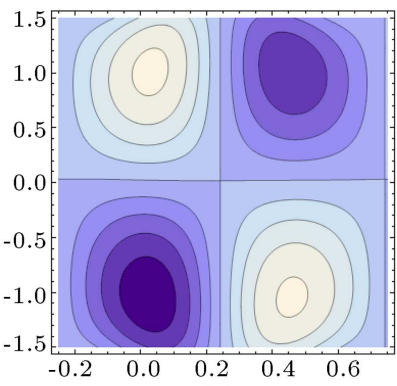

(a)

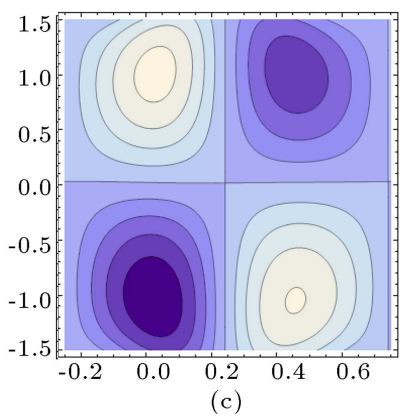

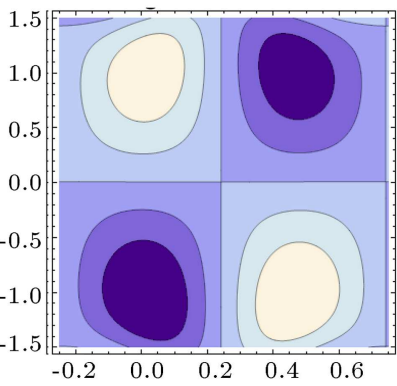

(b)

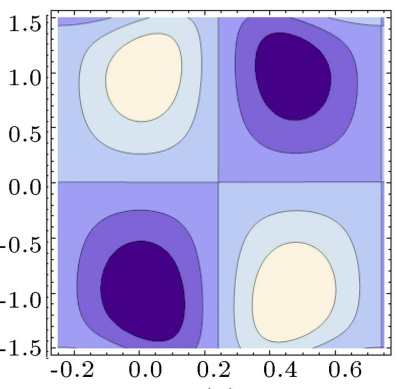

(d)
Figure 24. $\psi$ in terms of $M$ for (a) and (b) Maxwell model and (c) and (d) Hamilton-Crosser model when $E_{3}=0.01, t=0, E_{2}=0.01, \varepsilon=0.2, E_{1}=0.02, \phi=0.1$, $\mathrm{Gr}=0.03, \mathrm{Br}=3.0, \beta_{1}=0.01, \beta_{2}=-0.01, \gamma_{1}=0.01$, and $\gamma_{2}=-0.01$ : (a) and (c) $\mathrm{M}=1.0$ and (b) and (d) $\mathrm{M}=2.0$.

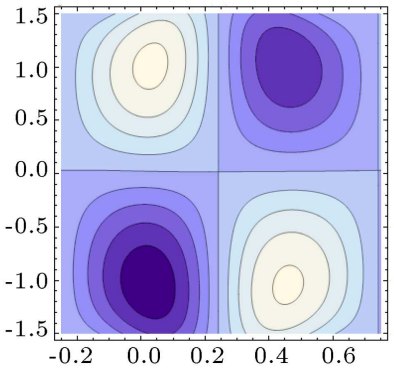

(a)

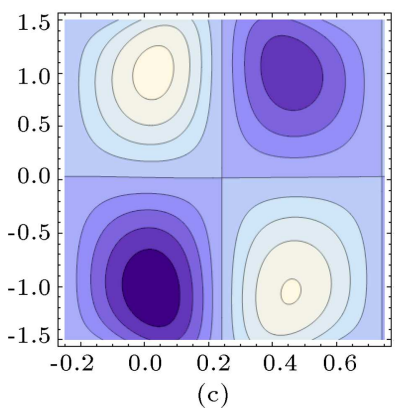

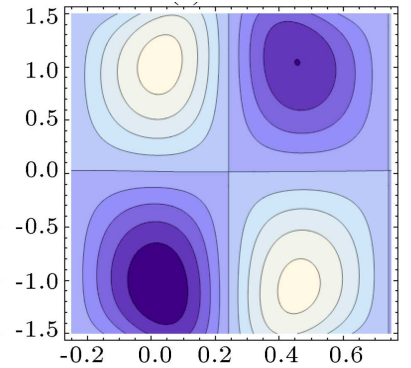

(b)

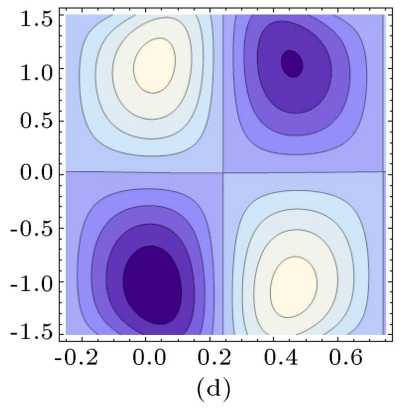

Figure 25. $\psi$ in terms of $\beta_{1}$ for (a) and (b) Maxwell model and (c) and (d) Hamilton-Crosser model when $E_{3}=0.01, t=0, E_{2}=0.01, \varepsilon=0.2, E_{1}=0.02, \phi=0.1$, $\mathrm{M}=1.0, \mathrm{Gr}=0.03, \mathrm{Br}=3.0, \beta_{2}=-0.01, \gamma_{1}=0.01$, and $\gamma_{2}=-0.01$ : (a) and (c) $\beta_{1}=0.01$, and (b) and (d) $\beta_{1}=0.03$

for the Maxwell model and in Figure 27(e)-(h) for the Hamilton-Crosser model. The models show the same behavior with these parameters, i.e. trapped bolus size

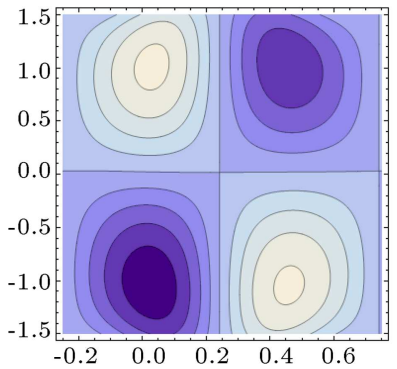

(a)

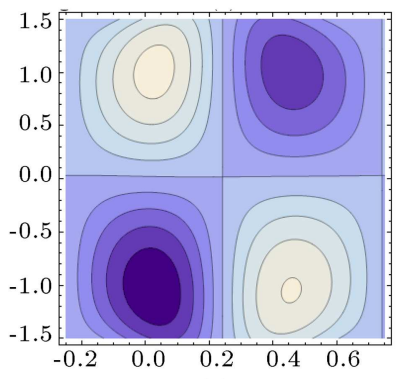

(c)

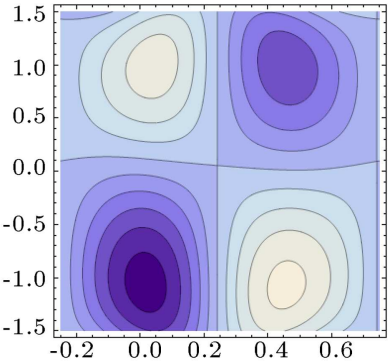

(b)

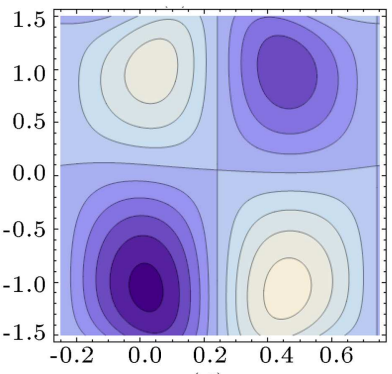

(d)
Figure 26. $\psi$ in terms of $\beta_{2}$ for (a) and (b) Maxwell model and (c) and (d) Hamilton-Crosser model when $E_{3}=0.01, t=0, E_{2}=0.01, \varepsilon=0.2, E_{1}=0.02, \phi=0.1$, $\mathrm{M}=1.0, \mathrm{Gr}=0.03, \mathrm{Br}=3.0, \beta_{1}=0.01, \gamma_{1}=0.01$, and $\gamma_{2}=-0.01$ : (a) and (c) $\beta_{2}=-0.01$; (b) and (d) $\beta_{2}=-0.03$.

increases with $E_{1}(=0.7,0.9)$ and $E_{2}(=0.4,0.6)$ and decreases with $E_{3}(=0.2,0.5)$.

\section{Conclusions}

The main conclusions drawn from the observations in this study may be summarized as follows:

- Enhancement in velocity was observed with both first-order and second-order velocity slips in Maxwell and Hamilton-Crosser models, while it was reduced with nanoparticle volume fraction;

- Values of the parameters were higher in HamiltonCrosser model than those in Maxwell model, especially near the center of the channel for velocity profile;

- Grashof and Hartman numbers had reverse effect on velocity;

- The quantities of temperature in Maxwell model exceeded those in Hamilton-Crosser model;

- Larger values of $\mathrm{Br} \Lambda^{-1}$ and Grashof number led to increase in entropy generation. On the other hand, Hartman number and nanoparticle volume fraction had an inverse impact on entropy generation;

- Bolus sizes increased by trapping phenomenon with both first- and second-order velocity slip parameters; 


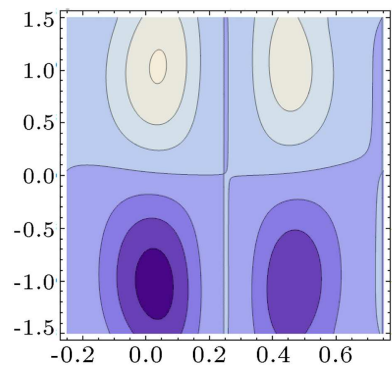

(a)

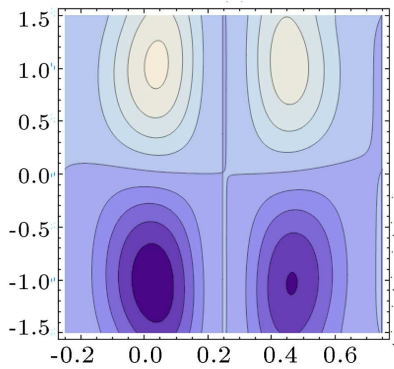

(c)

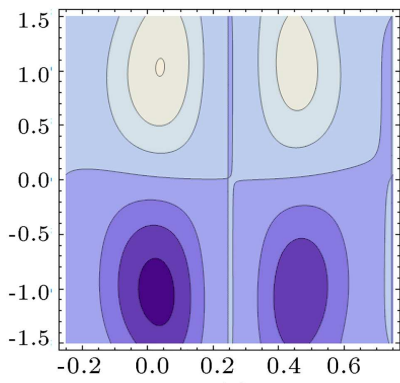

(e)

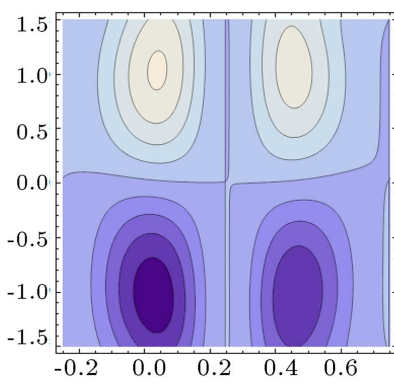

$(\mathrm{g})$

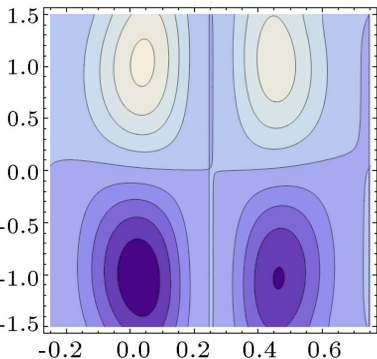

(b)

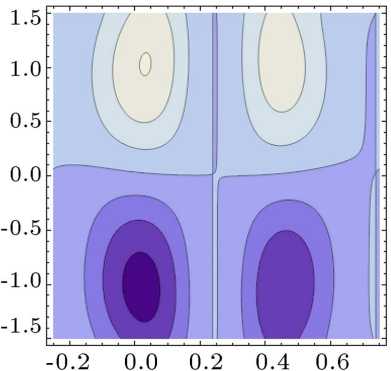

(d)

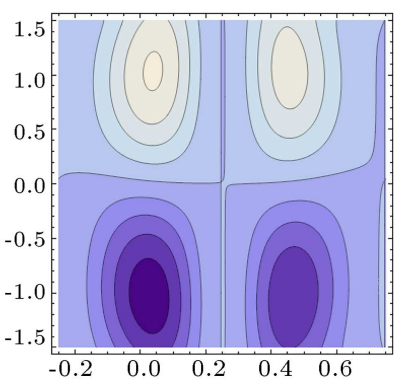

(f)

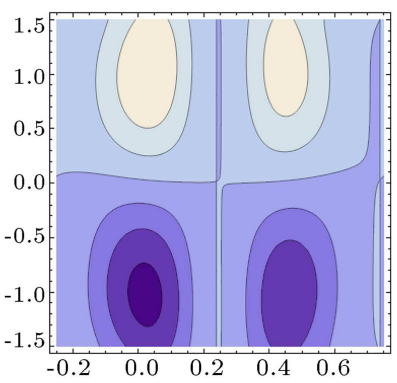

(h)
Figure 27. $\psi$ in terms of $E_{1}, E_{2}$, and $E_{3}$ for (a), (b), (c) and (d) Maxwell model and (e), (f), (g) and (h)

Hamilton-Crosser model when $t=0, \varepsilon=0.2, \phi=0.1$, $M=1.0, \mathrm{Gr}=0.03, \mathrm{Br}=3.0, \beta_{1}=0.01, \beta_{2}=-0.01$, $\gamma_{1}=0.01$, and $\gamma_{2}=-0.01$ : (a) and (e) $E_{1}=0.7$, $E_{2}=0.4$, and $E_{3}=0.2 ;(\mathrm{b})$ and (f) $E_{1}=0.9, E_{2}=0.4$, and $E_{3}=0.2 ;(\mathrm{c})$ and $(\mathrm{g}) E_{1}=0.7, E_{2}=0.6, E_{3}=0.2$; (d) and (h) $E_{1}=0.7, E_{2}=0.4$, and $E_{3}=0.5$.

- Bolus sizes were reduced with $E_{3}$ and increased with $E_{1}$ and $E_{2}$ in both Maxwell and Hamilton-Crosser models.

\section{Acknowledgement}

The authors are highly thankful to the Higher Education Commission (HEC) of Pakistan for finan- cial support of this work under project No. 203088/NRPU/R\&D/HEC/13.

\section{References}

1. Choi, S.U.S. "Enhancing thermal conductivity of the fluids with nanoparticles", ASME Fluids Eng. Div., 231, pp. 99-105 (1995).

2. Maxwell, J.C., A Treatise on Electricity and Magnetism, 2nd Edn., pp. 435-441 Oxford University Press, Cambridge (1904).

3. Hamilton, R.L. and Crosser, O.K. "Thermal conductivity of heterogeneous two component systems", $E C$ Fundam., 1, pp. 187-191 (1962).

4. Buongiorno, J. "Convective transport in nanofluids", ASME J. Heat Transfer, 128, pp. 240-250 (2006).

5. Mahanthesh, B., Gireesha, B.J., Animasaun, I.L., et al. "MHD flow of SWCNT and MWCNT nanoliquids past a rotating stretchable disk with thermal and exponential space dependent heat source", Phys. Scr., 94, 085214 (2019).

6. Hsiao, K.L. "Micropolar nanofluid flow with MHD and viscous dissipation effects towards a stretching sheet with multimedia feature", Int. J. Heat Mass Transfer, 112, pp. 983-990 (2017).

7. Makinde, O.D., Mahanthesh, B., Gireesha, B.J., et al. "MHD nanofluid flow past a rotating disk with thermal radiation in the presence of aluminum and titanium alloy nanoparticles", Defect Diffusion Forum, 384, pp. 69-79 (2018).

8. Hsiao, K.L. "Stagnation electrical MHD nanofluid mixed convection with slip boundary on a stretching sheet", Applied Thermal Engineering, 98, pp. 850-861 (2016).

9. Mahanthesh, B., Gireesha, B.J., Manjunatha, S., et al. "Effect of viscous dissipation and Joule heating on three-dimensional mixed convection flow of nano fluid over a non-linear stretching sheet in presence of solar radiation", J. Nanofluids, 6, pp. 735-742 (2017).

10. Hayat, T., Haider, F., Muhammad, T., et al. "Darcy-Forchheimer flow of carbon nanotubes due to a convectively heated rotating disk with homogeneous-heterogeneous reactions", J. Thermal Analysis Calorimetry, 137, pp. 1939-1949 (2019).

11. Hsiao, K.L. "To promote radiation electrical MHD activation energy thermal extrusion manufacturing system efficiency by using Carreau-nanofluid with parameters control method", Energy, 130, pp. 486-499 (2017).

12. Mahanthesh, B., Gireesha, B.J., Shehzad, S.A., et al. "Nonlinear radiated MHD flow of nanoliquids due to a rotating disk with irregular heat source and heat flux condition", Physica B, 537, pp. 98-104 (2018).

13. Abbasi, F.M., Hayat, T., Alsaadi, F., et al. "MHD peristaltic transport of spherical and cylindrical magneto-nanoparticles suspended in water", $A I P A d$ vances, 5, 077104 (2015). 
14. Latham, T.W. "Fluid motion in a peristaltic pump", MS Thesis, MIT, Cambridge, MA (1966).

15. Shapiro, A.H. "Pumping and retrograde diffusion in peristaltic waves", In Proc. Workshop Ureteral Reftm Children, Nat. Acad. Sci., Washington, DC, 1, pp. 109126 (1967).

16. Weinberg, S.L. "Theoretical and experimental treatment of peristaltic pumping and its relation to ureteral function", Ph.D. Thesis, MIT, Cambridge, MA (1970).

17. Srinivas, S. and Kothandapani, M. "Peristaltic transport in an asymmetric channel with heat transfer A note", Int. Commun. Heat Mass Transfer, 35, pp. 514-522 (2008).

18. Hina, S., Mustafa, M., Hayat, T., et al. "Peristaltic flow of Powell-Eyring fluid in curved channel with heat transfer: A useful application in biomedicine", Computer Methods Programs Biomedicine, 135, pp. 89-100 (2016).

19. Bhatti, M.M., Zeeshan, A., Ijaz, N., et al. "Mathematical modelling of nonlinear thermal radiation effects on EMHD peristaltic pumping of viscoelastic dusty fluid through a porous medium duct", Eng. Sci. Technol., Int. J., 20, pp. 1129-1139 (2017).

20. Shit, G.C. and Ranjit, N.K. "Role of slip velocity on peristaltic transport of couple stress fluid through an asymmetric non-uniform channel: Application to digestive system", J. Mol. Liq., 221, pp. 305-315 (2016).

21. Nawaz, S., Hayat, T., and Alsaedi, A. "Analysis of entropy generation in peristalsis of Williamson fluid in curved channel under radial magnetic field", Computer Methods Programs Biomedicine, 180, 105013 (2019).

22. Tripathi, D., Bhushan, S., Bég, O.A. "Unsteady viscous flow driven by the combined effects of peristalsis and electro-osmosis", Alex. Eng. J., 57, pp. 1349-1359 (2018).

23. Yasmeen, S., Asghar, S., Anjum, H.J., et al. "Analysis of Hartmann boundary layer peristaltic flow of Jeffrey fluid: Quantitative and qualitative approaches", Comm. Nonlinear Science Numerical Simulation, $\mathbf{7 6}$, pp. 51-65 (2019).

24. Hayat, T., Ayub, S., and Alsaedi, A. "Homogeneousheterogeneous reactions in curved channel with porous medium", Results Phys., 9, pp. 1455-1461 (2018).

25. Prakash, J., Sharma, A., and Tripathi, D. "Thermal radiation effects on electroosmosis modulated peristaltic transport of ionic nanoliquids in biomicrofluidics channel", J. Mol. Liqs., 249, pp. 843-855 (2018).

26. Hayat, T., Akram, J., Alsaedi, A., et al. "Endoscopy and homogeneous-heterogeneous reactions in MHD radiative peristaltic activity of Ree-Eyring fluid", Results Phys., 8, pp. 481-488 (2018).
27. Ellahi, R. and Hussain, F. "Simultaneous effects of MHD and partial slip on peristaltic flow of Jeffrey fluid in a rectangular duct", J. Magn. Magn. Mater., 393, pp. 284-292 (2015).

28. Hayat, T., Nawaz, S., Alsaedi, A., et al. "Mixed convective peristaltic flow of water based nanofluids with Joule heating and convective boundary conditions", PLoS ONE, 11, e0153537 (2016).

29. Akbar, N.S., Khan, L.A., Khan, Z.H., et al. "Natural propulsion with Lorentz force and nanoparticles in a bioinspired lopsided ciliated channel", J. B. Eng., 14, pp. $172-181$ (2017).

30. Abbasi, F.M., Gul, M., and Shehzad, S.A. "Hall effects on peristalsis of boron nitride-ethylene glycol nanofluid with temperature dependent thermal conductivity", Physica E: Low-dimensional Systems Nanostructures, 99, pp. 275-284 (2018).

31. Bhatti, M.M., Zeeshan, A., Ellahi, R., et al. "Mathematical modeling of heat and mass transfer effects on MHD peristaltic propulsion of two-phase flow through a Darcy-Brinkman-Forchheimer porous medium", $A d$ vanced Powder Technology, 29, pp. 1189-1197 (2018).

32. Akbar, N.S. and Butt, A.W. "Entropy generation analysis for the peristaltic flow of $\mathrm{Cu}$-water nanofluid in a tube with viscous dissipation", J. Hydrodynamics, 29, pp. 135-143 (2017).

33. Mosayebidorcheh, S. and Hatami, M. "Analytical investigation of peristaltic nanofluid flow and heat transfer in an asymmetric wavy wall channel", Int. J. Heat Mass Trans., 126, pp. 790-799 (2018).

34. Hayat, T., Nawaz, S., Alsaedi, A., et al. "Impact of second-order velocity and thermal slips in the mixed convective peristalsis with carbon nanotubes and porous medium", J. Mol. Liq., 221, pp. 434-442 (2016).

35. Bhatti, M.M., Zeeshan, A., Ellahi, R., et al. "Effects of coagulation on the two-phase peristaltic pumping of magnetized prandtl biofluid through an endoscopic annular geometry containing a porous medium", Chinese J. Physics, 58, pp. 222-234 (2019).

36. Abbasi, F.M. Hayat, T., and Ahmad, B. "Peristalsis of silver-water nanofluid in the presence of Hall and Ohmic heating effects: Applications in drug delivery", J. Mol. Liq., 207, pp. 248-255 (2015).

37. Bejan, A. "Second law analysis in heat transfer", Energy, 5, pp. 720-732 (1980).

38. Sheikholeslami, M. "New computational approach for exergy and entropy analysis of nanofluid under the impact of Lorentz force through a porous media", Computer Methods Applied Mechanics Engineering, 344, pp. 319-333 (2019).

39. Nawaz, S., Hayat, T., and Alsaedi, A. "Numerical study for peristalsis of Sisko nanomaterials with entropy generation", J. Thermal Analysis Calorimetry, 139, pp. 2129-2143 (2020). https://doi.org/10.1007/s10973-019-08546-5 
40. Hayat, T., Rafiq, M., Ahmad, B., et al. "Entropy generation analysis for peristaltic flow of nanoparticles in a rotating frame", Int. J. Heat Mass Trans., 108, pp. $1775-1786$ (2017).

\section{Biographies}

Tasawar Hayat is a Pakistani leading mathematician who has made pioneering research contributions to the area of fluid mechanics. He has received many awards for his numerous academic achievements. He is currently serving as a nationally distinguished professor at Quaid-i-Azam University, Pakistan.

Sadaf Nawaz is a $\mathrm{PhD}$ candidate in Mathematics at Quaid-i-Azam University, Pakistan. Her research interests are fluid mechanics, peristalsis, nanofluid, non-linear flow problems, entropy analysis, and heat transfer.

Ahmed Alsaedi is currently a professor at King Abdulaziz University, Saudi Arabia. He is in the list of leading mathematicians. His area of interests includes fluid dynamics and non-linear flow analysis. 